\title{
Inventário de Adiamento de Gratificação (DGI-35): Propriedades Psicométricas da Versão Brasileira
}

\author{
Gabriel Loureiro Figueira' \\ Vinícius do Carmo Oliveira de Lemos ${ }^{1}$ \\ Bruno Figueiredo Damásio ${ }^{1}$ \\ ${ }^{1}$ Universidade Federal do Rio de Janeiro
}

\begin{abstract}
Resumo
O Inventário de Adiamento de Gratificação (DGI-35) foi desenvolvido para avaliar os diferentes níveis de adiamento de gratificação presentes na população geral. O objetivo deste estudo foi apresentar as propriedades psicométricas do DGI-35 para a população brasileira e evidências de validade convergente com a Escala de Impulsividade de Barratt (BIS-11). Participaram 405 brasileiros (61,2\% mulheres), com idades entre 18 e 46 anos $(M=22,32, D P=4,78)$. Foram conduzidas análises fatoriais exploratórias e confirmatórias com vistas a avaliar a estrutura interna do instrumento. Dos cinco fatores originais, quatro tiveram estrutura interna semelhante à versão original, exceto o fator "Físico" que não se ajustou ao modelo, levando a criação de uma alternativa de 28 itens à escala original (DGI-28), que apresentou adequados índices de ajuste. Conforme esperado, o escore global da DGI-28, bem como os seus fatores estiveram negativamente relacionados ao escore global da BIS-11.

Palavras-chave: desvalorização pelo atraso, recompensa, comportamento impulsivo, psicometria, análise fatorial, testes psicológicos
\end{abstract}

The 35- Item Delaying Gratification Inventory (DGI-35): Psychometric Properties of the Brazilian Version

\begin{abstract}
The Delaying Gratification Inventory (DGI-35) was developed to evaluate different levels of gratification delaying in the general population. The purpose of this study was to present the psychometrics properties of the DGI-35 in the Brazilian population and evidence of convergent validity with the Barrat Impulsivity Scale (BIS-11). A total of 405 participants were selected (61.2\% women) aged between 18 and 46 years $(M=22.32, S D=4.78)$. Exploratory and confirmatory factor analyses were conducted to evaluate the internal structure of the instrument. Four out of five original factors showed identical consistency, except for the "Physical" factor that did not fit the model, leading to the creation of an alternative of 28 items to the original scale (DGI-28), which presented good fit indices. As expected, the global score of the DGI-28, as well as its factors were negatively correlated to the global score of the BIS-11.

Keywords: delay discounting; reward; impulsive behaviour; psychometrics; factor analysis; psychological tests;
\end{abstract}

\section{Inventario de Aplazamiento de Bonificación (DGI-35): Propiedades Psicométricas de la Versión Brasileña}

\section{Resumen}

El Inventario de Aplazamiento de Bonificación (DGI-35) fue desarrollado para evaluar los diferentes niveles de prórrogas de bonificación presentes en la población general. El objetivo de este estudio fue presentar las propiedades psicométricas del DGI-35 para la población brasileña y evidencias de validez convergentes con la Escala de Impulsividad de Barratt (BIS11). Participaron 405 brasileños (61,2\% mujeres) con edades entre 18 y 46 años $(M=22,32, D E=4,78)$. Fueron realizados análisis factorial confirmatoria y exploratorio que evaluaron la estructura interna del instrumento. De los cinco factores originales, cuatro tuvieron estructura interna semejante a la versión original, excepto el factor "Físico" que no se ajustó al modelo, creándose una alternativa de 28 ítems (DGI-28) a la escala original, la cual presentó índices de ajuste adecuados. Como se esperaba, la puntuación global de la DGI-28, así como sus factores estuvieron negativamente relacionados con la puntuación global de la BIS-11.

Palabras clave: devaluación por retraso; comportamiento impulsivo; psicometría; análisis factorial; tests psicológicos

\section{Introdução}

O adiamento de gratificação (AG), também conhecido como adiamento de recompensas (AR), é entendido como a capacidade de postergar gratificação imediata e persistir em comportamentos orientados a um objetivo, visando assim, resultados futuros (Mischel et al., 2011; Yu, Kam, \& Lee, 2016). A capacidade de adiar recompensas é compreendida por uma predileção individual na escolha de recompensas melhores e mais tardias frente à gratificação imediata, sendo necessária para guiar o comportamento na ausência de estímulos externos coercivos (Mischel, Shoda, \& Rodriguez, 1989).

É importante notificar que os termos "adiamento de gratificação" (delayed gratification), "adiamento de recompensas" (delayed rewards) e "desvalorização por atraso" (delay discouting) são utilizados como sinônimos 
da medição dos mesmos processos psíquicos pela literatura internacional (Rachlin, 2000). Apesar disso, há razões para crer que, apesar de altamente correlatos, dentro de um modelo de autorregulação do indivíduo e controle dos comportamentos impulsivos, tais termos se diferenciam. Adiamento de gratificação e adiamento de recompensas explicam a construção de comportamentos e estratégias para lidar com a saliência imediata percebida por uma recompensa, enquanto que a desvalorização por atraso seria uma habilidade mais complexa, relacionada a desvalorização subjetiva de uma recompensa mediante seu atraso (Reynolds \& Schiffbauer, 2005). Nesse artigo, optou-se por utilizar o termo "adiamento de gratificação" por ser o mais comumente reportado.

Indivíduos, em geral, variam na sua reatividade disposicional frente a recompensas (um mesmo estímulo é mais saliente a certos indivíduos) e no quanto é possível adiar gratificações. Esse desempenho em atividades de adiamento pode ser explicado, primordialmente, por mecanismos individuais e internalizados de um autocontrole eficiente (Blair, Peters, \& Granger, 2004; Duckworth, Tsukayama, \& Kirby, 2013).

Há um crescimento natural da capacidade em adiar recompensas nos seres humanos que pode ser explicado pelo desenvolvimento, durante a infância, de estratégias cognitivas orientadas a um objetivo, como autoverbalização, abstração, projeção temporal e controle da atenção (Sethi, Mischel, Aber, Shoda, \& Rodriguez, 2000). Embora esse crescimento ocorra de maneira similar entre sujeitos, a idade adulta será marcada por diferenças substanciais interindividuais nas habilidades de adiamento (Lee, Lan, Wang, \& Chiu, 2008).

O experimento mais utilizado para mensurar o AG, a "tarefa do marshmallow" (Mischel, 2014), apresenta um dilema envolvendo a escolha entre gratificar imediatamente ou postergar a gratificação em troca de um retorno superior àquele imediato. Essa técnica foi relacionada teoricamente à capacidade do sujeito em avaliar as consequências futuras de suas ações (Joireman, Balliet, Sprott, Spangenberg, \& Schultz, 2008; Stolarski, Ledzińska, \& Matthews, 2013). Consistia, originalmente, na exposição de uma criança a um estímulo positivo (e.g., um marshmallow) e, posteriormente, são apresentadas duas opções; consumir o doce imediatamente ou esperar brevemente (e.g., vinte minutos) sem comê-lo e ganhar mais doces como recompensa.

Embora amplamente disseminada, a tarefa do marshmallow apresenta limitações importantes. A utilização da observação comportamental como único critério para avaliar a distinção entre impulsos e autorregulação pode ser dúbia. Observando uma criança resistindo a um estímulo (e.g., negar sobremesa), é possível perceber que esta exerce um autocontrole consciente sobre os impulsos (e.g., "gostaria de comer o doce, mas não o farei”) em que há gasto energético de glicose e inibição ativa de comportamentos esperados (comer o doce), uma demonstração clara da ativação de funções de autorregulação. Porém, também é possível que a criança não esteja suficientemente salientada pela recompensa esperada (e.g., ela não gosta de doces) o que explicaria a ausência de necessidade de autorregulação efetiva. Portanto, comparar sujeitos apenas pela diferença no critério temporal do experimento não é fortuito a uma explicação concreta das diferenças individuais (Duckworth et al., 2013).

Apesar das suas limitações, o desempenho em tarefas de AG, como o teste do marshmallow tem sido associada à predição de sucesso ao longo do ciclo vital. Em um estudo longitudinal, iniciado com 185 crianças (103 meninas e 82 meninos com idade média de 4 anos e 4 meses), seguindo até o período da adolescência (idades entre 15 e 18 anos), foi demonstrado que sujeitos com maiores níveis de AG apresentaram maior sucesso individual e acadêmico, reportado por meio de avaliação parental, questionários de comportamentos e pelo desempenho em exames nacionais de educação (Shoda, Mischel, \& Peake, 1990). A capacidade de adiamento de gratificações também se demonstrou altamente consistente e estável, mesmo após longos períodos de até 40 anos (Casey et al., 2011). A habilidade de adiar gratificações teve, também, impacto no sucesso profissional e no bom relacionamento interpessoal na vida adulta (Newman, Caspi, Moffitt, \& Silva, 1997), assim como na sua capacidade preditiva do índice de massa corporal (Caleza, Yañez-Vico, Mendoza, \& Iglesias-Linares, 2016; Schlam, Wilson, Shoda, Mischel, \& Ayduk, 2013). Bembenutty e Karabenick (2004) demonstraram que o sucesso acadêmico é orientado, em certa medida, pela habilidade do estudante de resistir a tentações de gratificações imediatas e assim aumentar as chances de realizar mais objetivos a longo prazo.

Órgãos públicos de pesquisa em saúde (e.g., National Institute of Health, 2009) apresentam relatórios em que o AG mostra alto impacto na saúde pública. Níveis baixos de capacidade de AG apresentaram associação com maiores problemas sociais, abuso de substâncias, comportamentos sexuais de risco, psicopatologias, dívidas financeiras, criminalidade e baixa 
realização acadêmica (Abikoye \& Adekoya, 2010; Baumeister, Vohs, \& Tice, 2007; Bembenutty \& Karabenick, 2004; Dewall, Baumeister, Stillman, \& Gailliot, 2007; Gottdiener, Murawski, \& Kucharski, 2008; Moffitt et al., 2011; Seeyave et al., 2009).

Devido aos problemas do paradigma do marshmallow, alguns autores têm buscado investigar a capacidade de adiamento de gratificações por meio de escalas de autorrelato. Hoerger, Quirk e Weed (2011) propuseram um instrumento, denominado DGI-35, que hipotetiza cinco domínios do AG: comida, realização, interações sociais, dinheiro e prazeres físicos. Esta estrutura fatorial do fenômeno e o respectivo instrumento são frequentemente utilizados pela literatura mais recente (Baker \& Hoerger, 2012; Bartholdy et al., 2017; Giovanelli, Hoerger, Johnson, \& Gruber, 2013; Watson \& Milfont, 2017). Baumeister, Vohs e Tice (2007) também descrevem cinco domínios equivalentes aos anteriores, que compõem o AG. Esses domínios foram estudados individualmente por outros autores na literatura (Baumeister, Heatherton, \& Tice, 1994; Bembenutty \& Karabenick, 1998, 2004; Lee et al., 2008; Mischel et al., 1988; Ramanathan \& Williams, 2007). Atualmente, existem várias medidas de autorrelato para AG, como o Questionário de Adiamento de Gratificação (Ray \& Najman, 1986), a Escala Multidimensional de Adiamento de Gratificação (Ward, Perry, Woltz \& Doolin, 1989), a Escala Acadêmica de Adiamento de Gratificação (Bembenutty \& Karabenick, 1998) e o Inventário de Adiamento de Gratificação (Hoerger et al., 2011).

O Questionário de Adiamento de Gratificação (Ray \& Najman, 1986) é um instrumento de 12 itens e analisa o construto de forma unifatorial. Em sua conceitualização, essa medida elabora o fenômeno como uma medida global, não focalizado em situações-alvo ou domínios específicos de comportamentos associados a AG. Apesar de apresentar consistência interna dos itens satisfatória $(\alpha=0,72)$, apresentou correlações entre itens muito fraca $(r \cong 0,17)$. Ademais, o estudo original de validação não apresentou evidências de validade da escala com base em medidas externas e não apresentou índices de adequação de ajuste para o modelo.

A Escala Multidimensional de Adiamento de Gratificação (Ward et al., 1989) é um instrumento com 22 itens que hipotetiza o fenômeno com cinco fatores, porém a retenção fatorial por meio da análise exploratória apresenta apenas os domínios Sociopolítica e Realizações ( $\alpha=0,74$ e 0,68 , respectivamente), cuja variância explica $21 \%$ do construto. A correlação entre as facetas foi baixa $(r=0,21)$. Não foram apresentadas evidências de validade externa do instrumento. $\mathrm{O}$ instrumento também foi projetado para um público específico (estudantes, líderes sociais da comunidade negra universitária).

Outro breve questionário é a Escala Acadêmica de Adiamento de Gratificação (Bembenutty \& Karabenick, 1998), composta por 10 itens que também avalia o construto de forma unidimensional. $\mathrm{O}$ instrumento é voltado para o público acadêmico, apresentando o dilema do AG em situações dicotômicas da vida universitária. $\mathrm{O}$ instrumento apresentou boa consistência interna $(\alpha=0,77)$ e $47 \%$ de variância explicada do fenômeno. A análise fatorial confirmatória demonstrou que o índice de aderência do modelo foi baixo (GFI $=0,433)$. As correlações do instrumento com outras medidas utilizadas para validade também foram baixas: Deferment Gratification Questionnaire $(r=0,33)$, Impulsivity Scale $(r=-0,22)$, Nota Esperada do Aluno $(r=0,18)$ e Nota Final do Aluno $(r=0,13)$.

O Inventário de Adiamento de Gratificação (Delayed Gratification Inventory - DGI-35, Hoerger et al., 2011), por sua vez, apresenta boas propriedades psicométricas ( $\alpha=0,91$ em sua versão de 35 itens; $\alpha=$ 0,79 em sua versão de 10 itens) e uma proposição de cinco fatores: comida (capacidade de adiar recompensas por alimentos instigantes), realização (capacidade de adiar recompensas visando uma realização pessoal subjetiva, e.g., titulação acadêmica ou automelhoria), social (capacidade de adiar recompensas e suas eventuais relações de dependência ou impacto a terceiros), dinheiro (capacidade de postergar ações imediatas de recompensas financeiras visando benefício futuro, e.g., economizando ou investindo) e físico (capacidade de adiar ações por melhores desejos físicos e busca por sensações, e.g., sexo). Sua consistência interna foi boa $(\alpha=0,69-0,89)$ assim também como a correlação entre os fatores $(r=0,23-0,60)$.

Ainda, no estudo de validação (Hoerger et al., 2011), análises fatoriais confirmatórias apresentaram bons índices de ajuste para a estrutura de cinco fatores: ${ }_{\mathrm{s}-\mathrm{b}} \chi^{2}(550)=17,871, p<0,001$, e CFI $=0,964$, TLI $=0,962$; RMSEA $=0,057$; SRMR $=0,058$. Ademais, o estudo buscou validade externa com outros instrumentos de autorrelato submetidos aos participantes: Self-Control Scale $(r=0,71)$, Barrat Impulsivity Scale $(r=$ -0,66), Academic Delay of Gratification Scale $(r=0,51)$, Deferment of Gratification Questionnaire $(r=0,67)$. Tendo em vista que, no estudo de validação do instrumento, utilizaram-se quatro experimentos distintos, diversas 
escalas e extensos dados sociodemográficos, as propriedades aqui apresentadas foram expostas de forma mais sucinta.

Conforme demonstrado anteriormente, o Inventário de Adiamento de Gratificação (DGI-35) apresentou boas propriedades psicométricas, sendo considerado até o momento como uma ferramenta útil para avaliação do Adiamento de Gratificação. No Brasil, porém, ainda não existem pesquisas com o instrumento. Assim, o presente estudo buscou apresentar os procedimentos de adaptação e as propriedades psicométricas da DGI35 no contexto brasileiro. Este estudo faz parte de uma pesquisa maior, de caráter transcultural entre Brasil, Itália, China, Inglaterra e Estados Unidos, que tem por objetivo avaliar como países de culturas coletivistas e individualistas diferem em termos de características de apego, bem-estar, tomada de decisão e autorregulação em diferentes esferas da vida.

\section{Método}

\section{Participantes}

Participaram do estudo uma amostra composta por 405 brasileiros $(61,2 \%$ mulheres $)$ com idades entre 18 e 46 anos $(M=22,32, D P=4,78)$. Desse total, $54,1 \%$ eram solteiros, $37 \%$ estavam namorando e 5,9\% somavam os casados, noivos e morando juntos. Do total de participantes, $68,9 \%$ disseram possuir alguma religião ou crença espiritual, todos os participantes estavam cursando o ensino superior, sendo que entre estes $30,6 \%$ disseram trabalhar concomitantemente. Além disto, $87,9 \%$ da amostra afirmou que depende financeiramente de alguém. Trata-se de uma amostra por conveniência, de diferentes estados brasileiros, composta por $94,1 \%$ de participantes do RJ, 3\% de estados variados (AM, BA, DF, RS, RO e SP) e 2,9\% que não declararam seu estado de origem. Para participar do estudo, era necessário ser brasileiro e maior de idade.

\section{Instrumentos}

Inventário de Adiamento de Gratificação (DGI-35): O DGI-35 (Delaying Gratification Inventory; Hoerger et al., 2011). É composto por 35 itens (sete itens por domínio) respondidos em uma escala tipo Likert de cinco pontos, variando de 1 - Discordo totalmente a 5 - Concordo totalmente. O instrumento foi traduzido para o português brasileiro utilizando as diretrizes técnicas (Borsa, Damásio, \& Bandeira, 2012). A escala foi traduzida por dois tradutores independentes, sendo um brasileiro, fluente em língua inglesa, e um americano nativo, fluente na língua portuguesa. As duas traduções foram sintetizadas em uma única versão pela equipe de pesquisa, utilizando itens de ambas as traduções, que foram considerados mais adequados pelo time. Pequenos ajustes semânticos, idiomáticos e linguísticos foram realizados. Após a síntese das traduções e os referidos ajustes, a escala foi avaliada por um grupo de participantes, público-alvo $(n=12)$. Esses participantes responderam a uma ficha de avaliação da escala, que questionava sobre a compreensibilidade dos itens. Todos os itens foram considerados claros para todos os participantes, não havendo sugestão adicional de alterações.

Escala de Impulsividade de Barratt (BIS-11). A BIS-11 (Barrat Impulsivity Scale, versão 11; Patton, Stanford, \& Barratt, 1995, adaptada por Malloy-Diniz et al., 2010) é uma escala de autorrelato composta por 30 itens, respondidos em uma escala tipo Likert de quatro pontos, a saber: 1 = raramente ou nunca; 2 $=$ de vez em quando; $3=$ com frequência; $4=$ quase sempre/sempre. A escala avalia as manifestações da impulsividade em três domínios: impulsividade motora, impulsividade atencional e impulsividade por não planejamento, com base no modelo teórico proposto por Ernst Barratt (1959). Revisões sistemáticas recentes explicitaram as evidências de validade e precisão do instrumento e recomendam o seu uso para fins de pesquisa (Vasconcelos, Malloy-Diniz \& Correa, 2012). No presente estudo, foi utilizado o escore global da escala (Fidedignidade Composta $=0,907)$.

\section{Procedimentos Éticos e de Coleta de Dados}

A coleta de dados foi realizada virtualmente. $\mathrm{O}$ link para participação na pesquisa foi divulgado em redes sociais e por listas de e-mails. O estudo teve aprovação do Comitê de Ética do Centro de Filosofia e Ciências Humanas (CFCH) da Universidade Federal do Rio de Janeiro. O questionário continha o Termo de Consentimento Livre e Esclarecido (TCLE) de início e a pesquisa só prosseguiria mediante a concordância e aceite do participante. A privacidade dos respondentes, bem como o sigilo e a confidencialidade dos dados foram garantidas.

\section{Procedimentos de Análise dos Dados}

Inicialmente, foi realizada uma análise fatorial exploratória (AFE) com o objetivo de investigar a dimensionalidade da DGI-35. A AFE foi implementada por meio do método de estimação Weighted Least Squares Mean-and-Variance Adjusted (WLSMV; Muthén 
\& Muthén, 2015) no Programa Factor v. 10 (LorenzoSeva \& Ferrando, 2013). O número de fatores a ser retido foi estimado pela técnica da análise paralela com permutação aleatória dos dados (Timmerman \& Lorenzo-Seva, 2011).

Posteriormente, foi realizada uma análise fatorial confirmatória (AFC), implementada por meio do método de estimação Weighted Least Squares Mean-and-Variance Adjusted (WLSMV; Muthén \& Muthén, 2015). A AFC visou testar um modelo alternativo, obtido por meio da interpretação dos resultados da AFE. Foram usados o teste de qui-quadrado e a razão entre qui-quadrado e graus de liberdade $\left(\chi^{2} / \mathrm{g}\right)$; Comparative Fit Index (CFI), Tucker-Lewis Index (TLI) e o Root-Mean-Square Error of Approximation (RMSEA). De acordo com os critérios utilizados (Brown, 2014), a razão entre qui-quadrado e graus de liberdade $\left(\chi^{2} / g \emptyset\right)$ deve ser menor que 3; valores de RMSEA ( $90 \%$ IC) devem ser inferiores a 0,08 , com limite-superior do intervalo de confiança menor que 0,10 , e valores de CFI e TLI devem ser maiores que 0,90 , preferencialmente acima de 0,95 .

Índices de fidedignidade da escala foram avaliados por meio da fidedignidade composta, pois, em seu cálculo, é considerada a variação das cargas fatoriais dos itens, enquanto que, no coeficiente alfa, há o pressuposto da tau-equivalência, que determina que as cargas dos itens são iguais entre si - o que não se aplica neste estudo. Dessa forma, a FC apresenta-se como um indicador mais robusto de consistência interna (Valentini \& Damásio, 2017). A validade convergente da DGI foi avaliada com a Escala de Impulsividade de Barratt (Malloy-Diniz et al., 2010). É esperada uma correlação negativa e moderada entre as medidas.

\section{Resultados}

Inicialmente, buscou-se avaliar a dimensionalidade da DGI-35. Assim, foi executada uma AFE $(\mathrm{KMO}=$ 0,810; Teste de Esfericidade de Bartlett, $g l=595, p<$ $0,001)$. A análise paralela sugeriu cinco fatores como sendo o mais adequado para os dados (Ver Tabela 1). A Tabela 2 apresenta as cargas fatoriais dos itens.

Ao averiguar-se minunciosamente a disposição das cargas dos itens obtidas por meio da AFE, foi possível perceber que o Fator 5 (Físico) possui uma estrutura irregular. Apenas dois (DGI12 e DGI32), dos sete itens originais, apresentaram carga fatorial exclusivamente em sua dimensão originária, os demais itens se dispersaram em diferentes fatores. Especificamente, os itens
Tabela 1.

Análise Paralela DGI-35

\begin{tabular}{ccc}
\hline $\begin{array}{c}\text { Número de } \\
\text { fatores }\end{array}$ & $\begin{array}{c}\text { \% variância } \\
\text { explicada dos } \\
\text { dados reais }\end{array}$ & $\begin{array}{c}\text { \% variância } \\
\text { explicada dos } \\
\text { dados aleatórios }\end{array}$ \\
\hline 1 & 22,1 & 6,6 \\
2 & 11,5 & 6,1 \\
3 & 7,5 & 5,7 \\
4 & 7,1 & 5,4 \\
5 & 5,3 & 5,2 \\
6 & 4,3 & 5,0 \\
\hline
\end{tabular}

Nota. O número de fatores a ser retido é cinco, pois a partir desse número, a variância explicada dos dados aleatórios passa a ser maior do que a dos dados reais, sugerindo a retenção espúria de fatores.

DGI2, DGI7 e DGI22 carregaram no fator Social, o item DGI17 carregou no fator Realização e o item DGI27 no fator Comida. Esse resultado sugere que há uma confusão semântica dos itens que compõem o fator Físico, não avaliando explicitamente o comportamento que deveria (i.e., capacidade de adiar ações por melhores recompensas físicas e busca por sensações).

Conforme mencionado, os itens do fator Físico se agruparam de maneira desordenada nos outros fatores, sem que houvesse uma justificativa teórica para tal agrupamento. Então, foram avaliados diferentes modelos estruturais, via análises fatoriais confirmatórias (AFC) com vistas a encontrar uma solução adequada da DGI35 para o nosso estudo. Os modelos testados foram: 1) Modelo fatorial exploratório, composto por cinco fatores; 2) Modelo de cinco fatores, conforme obtido em Hoerger, Quirk e Weed, (2011); 3) Modelo de quatro fatores (agrupando os fatores físico e realização, conforme proposta de Hoerger et al., 2011); e 5) Modelo de quatro fatores, excluindo o fator Físico, derivado dos resultados dos modelos anteriores.

Conforme pode ser visto na Tabela 3, os modelos de cinco fatores (exploratório e original de Hoerger et al., 2011) não apresentaram índices de ajuste adequados, sendo, portanto, descartados. No estudo original de Hoerger et al., (2011), um modelo de quatro fatores é sugerido, agrupando os fatores Realização e Físico (F2 e F5), devido à sua alta correlação $(r=0,75)$, criando um fator mais abrangente que seria composto pelos 14 itens dos dois fatores. Apesar da alta correlação entre os fatores no estudo original não ter sido a mesma obtida nessa validação $(r=0,472)$, esse modelo 
Tabela 2.

Cargas Fatoriais da DGI-35 Separados pelos Fatores

\begin{tabular}{|c|c|c|c|c|c|}
\hline \multirow{3}{*}{ Itens } & \multicolumn{5}{|c|}{ Fatores } \\
\hline & F1 & $\mathrm{F} 2$ & F3 & F4 & F5 \\
\hline & Comida & Realização & Social & Dinheiro & Físico \\
\hline DGI1 & 0,779 & 0,092 & 0,051 & 0,251 & $-0,075$ \\
\hline DGI6 & 0,700 & 0,183 & 0,025 & 0,172 & 0,100 \\
\hline DGI11 & 0,623 & $-0,058$ & 0,016 & 0,213 & 0,103 \\
\hline DGI16 & 0,692 & 0,029 & 0,042 & 0,231 & $-0,233$ \\
\hline DGI21 & 0,456 & 0,122 & 0,193 & 0,197 & 0,271 \\
\hline DGI26 & 0,473 & 0,284 & 0,110 & 0,222 & 0,024 \\
\hline DGI31 & 0,492 & 0,020 & 0,135 & 0,206 & $-0,087$ \\
\hline DGI5 & $-0,059$ & 0,845 & 0,395 & 0,300 & $-0,057$ \\
\hline DGI10 & $-0,054$ & 0,868 & 0,328 & 0,211 & $-0,071$ \\
\hline DGI15 & 0,036 & 0,588 & 0,185 & 0,080 & 0,160 \\
\hline DGI20 & 0,122 & 0,727 & 0,272 & 0,199 & 0,218 \\
\hline DGI25 & 0,323 & 0,541 & 0,179 & 0,291 & 0,324 \\
\hline DGI30 & 0,107 & 0,523 & 0,238 & 0,218 & 0,139 \\
\hline DGI35 & 0,105 & 0,527 & 0,218 & 0,224 & 0,267 \\
\hline $\mathrm{DGI}^{\mathrm{a}}$ & $-0,011$ & 0,037 & 0,180 & $-0,049$ & 0,111 \\
\hline DGI8 & 0,007 & 0,289 & 0,802 & 0,196 & $-0,080$ \\
\hline DGI13 & $-0,022$ & 0,389 & 0,504 & 0,149 & $-0,113$ \\
\hline DGI18 & 0,055 & 0,383 & 0,861 & 0,196 & $-0,130$ \\
\hline DGI23 & 0,181 & 0,262 & 0,724 & 0,166 & 0,074 \\
\hline DGI28 & $-0,010$ & 0,316 & 0,482 & $-0,031$ & $-0,065$ \\
\hline DGI33 & 0,199 & 0,274 & 0,610 & 0,229 & 0,141 \\
\hline DGI4 & 0,183 & 0,335 & 0,257 & 0,743 & $-0,023$ \\
\hline DGI9 & 0,393 & 0,074 & 0,054 & 0,635 & 0,198 \\
\hline DGI14 & 0,237 & 0,315 & 0,325 & 0,736 & 0,026 \\
\hline DGI19 & $-0,006$ & 0,309 & 0,173 & 0,431 & 0,140 \\
\hline DGI24 & 0,318 & 0,225 & 0,157 & 0,792 & 0,376 \\
\hline DGI29 & 0,212 & 0,258 & 0,221 & 0,782 & 0,019 \\
\hline DGI34 & 0,319 & 0,207 & 0,114 & 0,776 & 0,377 \\
\hline DGI2 & 0,076 & 0,040 & 0,367 & 0,180 & 0,196 \\
\hline DGI7 & $-0,112$ & 0,289 & $0,572 *$ & 0,148 & 0,425 \\
\hline DGI12b & 0,106 & 0,226 & 0,040 & 0,156 & 0,300 \\
\hline DGI17 & 0,137 & $0,563^{*}$ & 0,289 & 0,251 & 0,070 \\
\hline DGI22 & $-0,008$ & 0,135 & $0,446 *$ & 0,149 & 0,606 \\
\hline DGI27 & $0,481 *$ & 0,250 & 0,082 & 0,278 & 0,256 \\
\hline DGI32 & 0,292 & 0,244 & 0,075 & 0,129 & 0,405 \\
\hline
\end{tabular}

Nota. ${ }^{\mathrm{a}, \mathrm{b}}$ Os itens em questão não apresentam carga fatorial acima de 0.4. As cargas fatoriais em negrito estão adequadas em seu fator teoricamente esperado. As cargas fatoriais notadas com um asterisco estão fora da conceitualização teórica e são significativas $(\boldsymbol{\lambda}>0,40)$. 
Tabela 3.

Análise Fatorial de Diferentes Modelos da DGI

\begin{tabular}{lccccc}
\hline Modelo & $\chi^{2}(g l)$ & $\chi^{2} / g l$ & CFI & TLI & RMSEA $(90 \%$ IC) \\
\hline $\begin{array}{l}\text { Cinco fatores } \\
\text { (AFE) }\end{array}$ & $1026,098(430)$ & 2,39 & 0,918 & 0,886 & $0,059(0,055-0,064)$ \\
$\begin{array}{l}\text { Cinco fatores Original } \\
\text { (AFC) }\end{array}$ & $1518,072(550)$ & 2,76 & 0,867 & 0,856 & $0,067(0,063-0,071)$ \\
$\begin{array}{l}\text { Quatro fatores F2+F5 } \\
\text { (AFC) }\end{array}$ & $1613,040(554)$ & 2,91 & 0,854 & 0,843 & $0,069(0,066-0,073)$ \\
DGI-28 (AFC) & $886,410(344)$ & 2,57 & 0,918 & 0,910 & $0,063(0,058-0,068)$ \\
\hline
\end{tabular}

(agrupando os fatores Realização - F2 e Físico - F5 em um único fator) também foi testado (ver tabela 3). Os índices de ajuste desse modelo de quatro fatores também não foram adequados, corroborando os resultados encontrados por Hoerger et al., (2011).

Por fim, uma vez que o fator 5 (Físico) e seus respectivos itens não se ajustaram a nenhum dos modelos apresentados, optou-se por excluí-lo totalmente. Assim, foi avaliado um terceiro modelo (ver tabela 3), considerando quatro fatores, agora intitulado DGI-28. A DGI-28 (ver Figura 1), composta por quatro fatores, foi o único modelo a obter índices de ajuste satisfatórios: $\chi^{2}=886.410, p<0,01 ; \chi^{2} / g l=2.57$; CFI $=0,918$, $\mathrm{TLI}=0,910, \mathrm{RMSEA}=0,063(\mathrm{IC} 90 \% 0,052-0,068) \mathrm{e}$, portanto, aquele que melhor representa o instrumento na validação brasileira.

Buscando evidências de validade convergente da DGI-28, foram feitas análises de correlação com a BIS-11. A Tabela 4 demonstra as correlações entre os fatores do instrumento DGI-28 proposto e a nota global da BIS-11. Conforme esperado, as relações entre a DGI-28 e a BIS-11 foram negativas, demonstrando que os índices de impulsividade se correlacionaram negativamente com o AG.

\section{Discussão}

O presente estudo teve por objetivo avaliar as propriedades psicométricas da versão brasileira da DGI-35. Inicialmente, AFEs sugeriram cinco fatores para os dados. Apesar disso, ao submeter o nosso modelo à uma AFC, os valores de aderência foram insatisfatórios. Isso se deu primordialmente por conta de o fator Físico possuir propriedades psicométricas muito destoantes do restante do instrumento. Esse fenômeno da discrepância no domínio físico também foi relatado no instrumento original (Hoerger et al., 2011): em ambos os estudos o fator Físico possui os itens com as cargas fatoriais com valores muito inferiores ao restante $\mathrm{e}$ índices insatisfatórios de consistência interna $(\alpha=0,69$ e $\mathrm{FC}=0,456$ ).

Foram realizadas análises fatoriais confirmatórias (AFCs), buscando avaliar outras estruturas existentes na literatura. Entretanto, para os dados brasileiros, nenhuma dessas estruturas se mostrou adequada. Evidências psicométricas que sugerissem a plausibilidade do modelo com cinco fatores não foram encontradas. Do ponto de vista teórico, a literatura apresenta que a estrutura do fator Físico (DGI2, DGI7, DGI12, DGI17, DGI22, DGI27 e DGI32) se correlaciona com a busca por sensações, neuroticismo, ansiedade, comportamentos de risco, pensamentos sexuais frequentes, propensão a quebrar regras e baixo controle dos impulsos (Hoerger et al., 2011). Acredita-se que isso fora alcançado no conteúdo textual dos itens adaptados para o contexto brasileiro (para a lista completa dos itens adaptados ver Anexo A).

Do ponto de vista psicométrico, espera-se que itens que fazem parte de um fator, tenham elevada carga fatorial (valores acima de 0,3 e 0,4) preferencialmente apenas em um fator. Assim como no instrumento original, esses valores não foram encontrados para o fator Físico. Dessa maneira, fez-se necessário avaliar, item a item, as razões que possivelmente justificariam teoricamente e psicometricamente a remoção dessa faceta.

Os itens DGI2 ("Consigo controlar meus desejos sexuais"), DGI7 (“Antes de ter uma relação sexual, prefiro conhecer melhor a pessoa"), DGI22 ("Prefiro explorar de imediato o lado físico das relações amorosas"), desse fator, apresentaram carga fatorial cruzada 


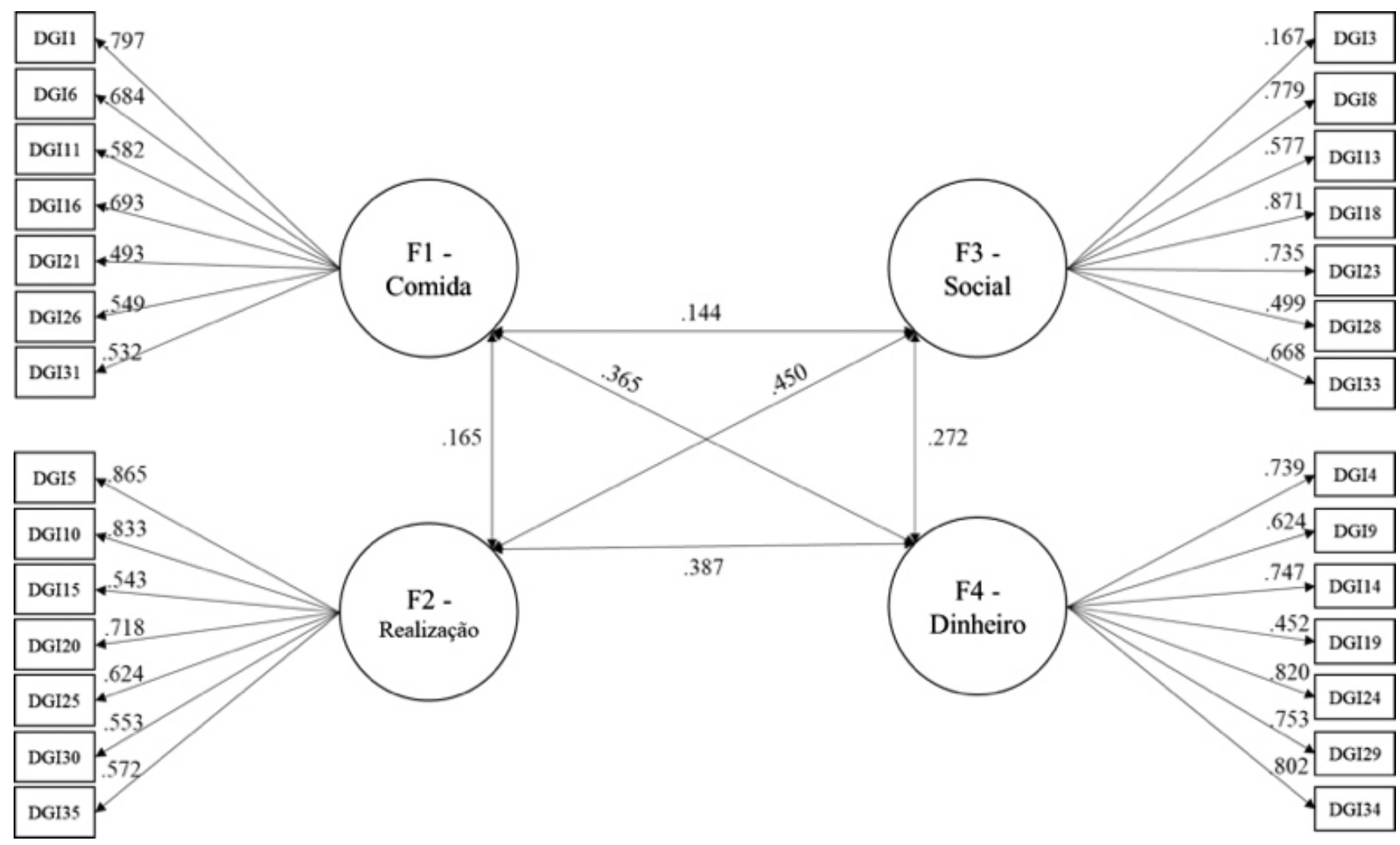

Figura 1. Modelo ilustrativo da versão de quatro fatores da DGI-28.

Tabela 4.

Tabela de Correlacão entre DGI-28 e BIS-11

\begin{tabular}{lcccccc}
\hline Rô de Spearman & BIS11 & DGI28 & F1 - Comida & F2 - Realização & F3 - Social & F4 - Dinheiro \\
\hline BIS-11 & - & & & & \\
DGI-28 & $-0,620^{* *}$ & - & & & \\
F1 - Comida & $-0,381^{* *}$ & $0,648^{* *}$ & - & & \\
F2 - Realização & $-0,524^{* *}$ & $0,661^{* *}$ & $0,144^{* *}$ & - & - \\
F3 - Social & $-0,231^{* *}$ & $0,525^{* *}$ & $0,111^{*}$ & $0,343^{* *}$ & - & \\
F4 - Dinheiro & $-0,484^{* *}$ & $0,711^{* *}$ & $0,286^{* *}$ & $0,322^{* *}$ & $0,201 * *$ & - \\
\hline
\end{tabular}

Nota.** A correlação é significativa no nível 0,01 (2 extremidades).

*. A correlação é significativa no nível 0,05 (2 extremidades).

no fator F3 (Social). Isso pode ter acontecido devido ao conteúdo semântico dos itens citados ser semelhante (sexualidade, sexualização) e estar fortemente associado a atividades ou afetos compartilhados com outros indivíduos (por essa razão, talvez, houve o carregamento no fator Social). Apesar disso, os itens do fator Social, não apresentam em sua redação e em seu sentido semântico qualquer referência a sexualidade, sexualização ou relações sexuais, o que reforça que não há razão psicométrica (por conta da carga fatorial cruzada) e nem teórica (pelo conteúdo semântico do fator Social ser diferente do fator Físico) para justificar a 
permanência dos itens no instrumento. Apesar do imaginário popular sobre a sexualidade brasileira endossar uma ideia de que a socialização visa os fins sexuais, ou de que a população abertamente discute questões sobre a temática, empiricamente essas afirmativas não são corroboradas (Heilborn, 2006). Por essa razão, esses itens foram removidos da versão final refinada.

O item DGI17 do fator Físico ("Eu já deixei de lado prazeres físicos ou conforto para alcançar meus objetivos") apresentou carga fatorial no fator F2, relacionado à Realização. A carga fatorial do item foi de 0,070 no fator Físico e de 0,563 no fator Realização. É possível que a redação do item tenha sido ambígua, incluindo dois conteúdos distintos (1) deixar de lado prazer físico ou conforto e (2) alcançar objetivos, causando nos respondentes duas possibilidades de resposta e, no caso do Brasil, enfatizando o segundo conteúdo, justificando a alta carga fatorial do item no fator Realização. Tendo em vista esse problema de carga inadequada do item no fator correto e a remoção anterior de três dos sete itens que compunham o fator, optou-se por remover também esse item do instrumento final refinado, não fazendo sentido adicioná-lo ao fator Realização, o que violaria a sua estrutura original.

Por fim, o item DGI27 ("Sempre adio cumprir uma tarefa que exige muito esforço físico") apresentou carga fatorial no fator Comida superior a sua carga no fator original (no fator Comida $=0,481 \mathrm{e}$ no fator Físico $=0,256)$ o que aliado ao conteúdo semântico do item pode indicar uma interpretação de que o esforço físico, nesse caso, estaria associado à comida (seja pelo viés da resistência a comer guloseimas ser um esforço físico tanto quanto pelo viés do esforço físico de bem-estar e saúde estar associado a uma alimentação saudável e restrita). Apesar dessa especulação por parte dos autores, não parece haver nenhuma explicação mais consistente que justifique a permanência do item no instrumento final. Uma vez que quatro dos cinco itens que compunham o fator original Físico já haviam sido removidos, optou-se por remover também esse item anteriormente citado. Os dois itens que sobraram no fator Físico (DGI12 e DGI32) foram também excluídos. O DGI12 apresenta baixa carga fatorial $(\boldsymbol{\lambda}=0,30)$; e o item DGI32 ficou isolado nesse fator, o que o torna inexistente.

A DGI-28 foi o único modelo que apresentou índices de ajuste aceitáveis. Assim, essa versão foi considerada a versão final da escala. Para fins de validade convergente, a DGI-28 foi correlacionada com a BIS11 , pois ambas são medidas de autorrelato que avaliam o autocontrole do indivíduo. Esse procedimento também foi realizado no estudo original de validação do instrumento e valores semelhantes de correlação entre os instrumentos foram encontrados $(r=-0,66$ no estudo original e $r=-0,62$ no presente estudo). Os resultados demonstraram que, como esperado, indivíduos que possuem maiores índices no adiamento de gratificações o fazem por meio de um complexo sistema cognitivo de autorregulação que inclui um maior controle sobre a impulsividade (Baumeister et al., 2007). O controle da impulsividade compõe parte dos processos realizados no AG (Mischel, 1974) e ambos fundamentam prerrogativas da autorregulação na tomada de decisão e percepção do tempo nos indivíduos (Wittmann \& Paulus, 2008).

\section{Considerações Finais}

O presente estudo teve por objetivo apresentar evidências de validade do Inventário de Adiamento de Gratificação para o contexto brasileiro. Conforme foi demonstrado, a escala apresentou adequadas propriedades psicométricas após a retirada de um fator (Físico). Uma vez que a amostra desse estudo não é representativa da população brasileira, não é possível garantir que essa estrutura fatorial se comportará de maneira semelhante em futuros estudos. É importante ressaltar que não existem justificativas teóricas que argumentem por que o construto AG seria composto pelas respectivas facetas que compõem esse instrumento (físico, comida, realização e social). Assim, não é sabido o quanto a remoção do fator físico prejudica a mensuração do fenômeno. Futuros estudos utilizando a versão completa da DGI-35 poderão esclarecer em que medida o fator Físico, de fato, não é plausível na população brasileira. Por essa razão, o Anexo A apresenta os itens adaptados do fator Físico removidos da estrutura final. Considerando a importância do construto AG em diversos desfechos ao longo do ciclo vital, pesquisadores e demais interessados poderão se beneficiar do uso desse instrumento em suas práticas profissionais.

\section{Referências}

Abikoye, G. E., \& Adekoya, J. A. (2010). Predicting substance abuse in a sample of Nigerian undergraduate students: The Role of Core Self-evaluations and Delay of Gratification. Psychological Studies, 55(4), 299-307. doi: 10.1007/s12646-010-0047-9 
Baker, C. N., \& Hoerger, M. (2012). Parental childrearing strategies influence self-regulation, socio-emotional adjustment, and psychopathology in early adulthood: Evidence from a retrospective cohort study. Personality and Individual Differences, 52(7), 800-805. doi:10.1016/j.paid.2011.12.034

Barratt, E. S. (1959). Anxiety and impulsiveness related to psychomotor efficiency. Perceptual and motor skills, 9(3), 191-198. doi:10.2466/pms.1959.9.3.191

Bartholdy, S., Rennalls, S., Danby, H., Jacques, C., Campbell, I. C., Schmidt, U., \& O’Daly, O. G. (2017). Temporal Discounting and the Tendency to Delay Gratification across the Eating Disorder Spectrum. European Eating Disorders Review. doi:10.1002/ erv.2513

Baumeister, R. F., Heatherton, T. F., \& Tice, D. M. (1994). Losing control: How and why people fail at selfregulation. Cambridge, Massachusetts: Academic Press.

Baumeister, R. F., Vohs, K. D., \& Tice, D. M. (2007). The Strength Model of Self-Control. Current Directions in Psychological Science, 16(6), 351-355. doi: 10.1111/j.1467-8721.2007.00534.x

Bembenutty, H., \& Karabenick, S. A. (1998). Academic delay of gratification. Learning and Individual Differences, 10(4), 329-346. doi: 10.1016/ S1041-6080(99)80126-5

Bembenutty, H., \& Karabenick, S. A. (2004). Inherent Association Between Academic Delay of Gratification, Future Time Perspective, and Self-Regulated Learning. Educational Psychology Review, 16(1), 35-57. doi: 10.1023/B:EDPR.0000012344.34008.5c

Blair, C., Peters, R., \& Granger, D. (2004). Physiological and neuropsychological correlates of approach/ withdrawal tendencies in preschool: Further examination of the behavioral inhibition system/ behavioral activation system scales for young children. Developmental Psychobiology, 45(3), 113-124. doi: 10.1002/dev.20022

Borsa, J. C., Damásio, B. F., \& Bandeira, D. R. (2012). Adaptação e validação de instrumentos psicológicos entre culturas: Algumas considerações. Paidéia, 22(53). doi: 10.1590/1982-43272253201314

Brown, T. A. (2014). Confirmatory factor analysis for applied research. New York City, New York: The Guilford Press.
Caleza, C., Yañez-Vico, R. M., Mendoza, A., \& IglesiasLinares, A. (2016). Childhood obesity and delayed gratification behavior: A systematic review of experimental studies. Journal of Pediatrics. Mosby. doi: 10.1016/j.jpeds.2015.10.008

Casey, B. J., Somerville, L. H., Gotlib, I. H., Ayduk, O., Franklin, N. T., Askren, M. K., ... Shoda, Y. (2011). Behavioral and neural correlates of delay of gratification 40 years later. Proceedings of the National Academy of Sciences of the United States of America, 108(36), 14998-15003. doi: 10.1073/ pnas. 1108561108

Dewall, C., Nathan, Baumeister, R. F., Stillman, T. F., \& Gailliot, M. T. (2007). Violence restrained: Effects of self-regulation and its depletion on aggression. Journal of Experimental Social Psychology, 43, 62-76. doi: 10.1016/j.jesp.2005.12.005

Duckworth, A. L., Tsukayama, E., \& Kirby, T. A. (2013). Is it really self-control? Examining the predictive power of the delay of gratification task. Personality \& social psychology bulletin, 39(7), 843-855. doi: 10.1177/0146167213482589

Giovanelli, A., Hoerger, M., Johnson, S. L., \& Gruber, J. (2013). Impulsive responses to positive mood and reward are related to mania risk. Cognition \& Emotion, 27(6), 1091-1104. doi: 10.1080/02699931.2013.772048

Gottdiener, W. H., Murawski, P., \& Kucharski, L. T. (2008). Using the Delay Discounting Task to test for failures in ego control in substance abusers: A meta-analysis. Psychoanalytic Psychology, 25(3), 533549. doi: 10.1037/0736-9735.25.3.533

Heilborn, M. L. (2006). Entre as tramas da sexualidade brasileira. Estudos Feministas, 14(336). doi: 10.1590/ S0104-026X2006000100004

Hoerger, M., Quirk, S. W., \& Weed, N. C. (2011). Development and validation of the Delaying Gratification Inventory. Psychological assessment, 23(3), 725-738. doi: $10.1037 /$ a0023286

Joireman, J., Balliet, D., Sprott, D., Spangenberg, E., \& Schultz, J. (2008). Consideration of future consequences, ego-depletion, and self-control: Support for distinguishing between CFC-Immediate and CFC-Future sub-scales. Personality and Individual Differences, 45(1), 15-21. doi: 10.1016/j. paid.2008.02.011 
Lee, P. L., Lan, W., Wang, C. L., \& Chiu, H. Y. (2008). Helping Young Children to Delay Gratification. Early Childhood Education Journal, 35(6), 557-564. doi: 10.1007/s10643-008-0240-9

Lorenzo-Seva, U., \& Ferrando, P. J. (2013). FACTOR 9.2: A Comprehensive program for fitting exploratory and semiconfirmatory factor analysis and IRT models. Applied Psycbological Measurement, 37(6), 497-498. doi: 10.1177/0146621613487794

Malloy-Diniz, L. F., Mattos, P., Leite, W. B., Abreu, N., Coutinho, G., Paula, J. J. D., ... \& Fuentes, D. (2010). Tradução e adaptação cultural da Barratt Impulsiveness Scale (BIS-11) para aplicação em adultos brasileiros. Jornal Brasileiro de Psiquiatria, 59(2), 99-105. doi: 10.1590/ S0047-20852010000200004

Mischel, W. (1974). Processes in delay of gratification. Em Berkowitz, L. (Org.) Advances in experimental social psychology (Vol. 7, pp. 249-292). New York City, New York: Academic Press.

Mischel, W. (2014). The Marshmallow Test: Understanding Self Control and how to Master it. New York City, New York: Random House.

Mischel, W., Ayduk, O., Berman, M. G., Casey, B. J., Gotlib, I. H., Jonides, J., ... Shoda, Y. (2011). "Willpower" over the life span: Decomposing selfregulation. Social Cognitive and Affective Neuroscience, 6(2), 252-256. doi: 10.1093/scan/nsq081

Mischel, W., Shoda, Y., \& Peake, P. K. (1988). The nature of adolescent competencies predicted by preschool delay of gratification. Journal of Personality and Social Psychology, 54(4), 687-696. doi:10.1037/0022-3514.54.4.687

Mischel, W., Shoda, Y., \& Rodriguez, M. I. (1989). Delay of gratification in children. Science, 244(4907), 933938. doi: $10.1126 /$ science. 2658056

Moffitt, T. E., Arseneault, L., Belsky, D., Dickson, N., Hancox, R. J., Harrington, H., ... Caspi, A. (2011). A gradient of childhood self-control predicts health, wealth, and public safety. Proceedings of the National Academy of Sciences of the United States of America, 108(7), 2693-2698. doi: 10.1073/ pnas. 1010076108

Muthén, L. K., \& Muthén, B. (2015). Mplus. The comprehensive modelling program for applied researchers: user's guide, 5.
National Institutes of Health. (2009). NIH science of behavior change. In Meeting Summary, Bethesda, MD (pp. 1-39).

Newman, D. L., Caspi, A., Moffitt, T. E., \& Silva, P. A. (1997). Antecedents of adult interpersonal functioning: Effects of individual differences in age 3 temperament. Developmental Psychology, 33(2), 206. doi: 10.1037/0012-1649.33.2.206

Patton, J. H., Stanford, M. S., \& Barratt, E. S. (1995). Factor structure of the Barratt impulsiveness scale. Journal of Clinical Psychology, 51(6), 768-774. doi: 10.1002/1097-4679(199511)51:6<768::AIDJCLP2270510607>3.0.CO;2-1

Rachlin, H. (2000). The science of self-control. Cambridge, Massachusetts: Harvard University Press.

Ramanathan, S., \& Williams, P. (2007). Immediate and delayed emotional consequences of indulgence: The Moderating Influence of Personality Type on Mixed Emotions. Journal of Consumer Research, 34(2), 212-223. doi: 10.1086/519149

Ray, J. J., \& Najman, J. M. (1986). The Generalizability of Deferment of Gratification. The Journal of Social Psychology, 126(1), 117-119. doi: 10.1080/00224545.1986.9713578

Reynolds, B., \& Schiffbauer, R. (2005). Delay of gratification and delay discounting: A Unifying Feedback Model of Delay-Related Impulsive Behavior. The Psychological Record, 55(3), 439-460. doi: 10.1007/ BF03395520

Schlam, T. R., Wilson, N. L., Shoda, Y., Mischel, W., \& Ayduk, O. (2013). Preschoolers' delay of gratification predicts their body mass 30 years later. Journal of Pediatrics, 162(1), 90-93. doi: 10.1016/j. jpeds.2012.06.049

Seeyave, D. M., Coleman, S., Appugliese, D., Corwyn, R. F., Bradley, R. H., Davidson, N. S., ... Lumeng, J. C. (2009). Ability to Delay Gratification at Age 4 Years and Risk of Overweight at Age 11 Years. Archives of Pediatrics \& Adolescent Medicine, 163(4), 303. doi: $10.1001 /$ archpediatrics. 2009.12

Sethi, A., Mischel, W., Aber, J. L., Shoda, Y., \& Rodriguez, M. L. (2000). The role of strategic attention deployment in development of self-regulation: Predicting preschoolers' delay of gratification from mothertoddler interactions. Developmental Psychology, 36(6), 767-777. doi: 10.1037/0012-1649.36.6.767 
Shoda, Y., Mischel, W., \& Peake, P. K. (1990). Predicting adolescent cognitive and self-regulatory competencies from preschool delay of gratification: Identifying diagnostic conditions. Developmental Psychology, 26(6), 978-986. doi: 10.1037/0012-1649.26.6.978

Stolarski, M., Ledzińska, M., \& Matthews, G. (2013). Morning is tomorrow, evening is today: relationships between chronotype and time perspective. Biological Rhythm Research, 44(2), 181-196. doi: 10.1080/09291016.2012.656248

Timmerman, M. E., \& Lorenzo-Seva, U. (2011). Dimensionality assessment of ordered polytomous items with parallel analysis. Psychological Methods, 16(2), 209. doi: 10.1037/a0023353

Valentini, F., \& Damásio, B. F. (2017). Variância média extraída e confiabilidade composta: Indicadores de precisão. Psicologia: teoria e pesquisa, 32(2). doi: 10.1590/0102-3772e322225.

Vasconcelos, A. G., Malloy-Diniz, L., Correa, H., \& Gomide Vasconcelos, A. (2012). Systematic review of psychometric proprieties of Barratt Impulsiveness
Scale Version 11 (BIS-11). Clinical Neuropsychiatry, 9(2). Recuperado de http://psycnet.apa.org/ record/2012-14979-003

Ward, W. E., Perry, T. B., Woltz, J., \& Doolin, E. (1989). Delay of gratification among black college student leaders. Journal of Black Psychology, 15(2), 111-128. doi: 10.1177/00957984890152004

Watson, S. J., \& Milfont, T. L. (2017). A short-term longitudinal examination of the associations between self-control, delay of gratification and temporal considerations. Personality and Individual Differences, 106, 57-60. doi: 10.1016/j.paid.2016.10.023

Wittmann, M., \& Paulus, M. P. (2008). Decision making, impulsivity and time perception. Trends in cognitive sciences, 12(1), 7-12. doi: 10.1016/j.tics.2007.10.004

Yu, J., Kam, C.-M., \& Lee, T. M. C. (2016). Better Working Memory and Motor Inhibition in Children Who Delayed Gratification. Frontiers in psychology, 7, 1098. doi: 10.3389/ fpsyg.2016.01098

Recebido em: 12/10/2017 Reformulado em: 19/06/2018, 08/10/2018 Aceito: 08/02/2019 


\section{Anexo A}

Lista de itens da DGI-35

\begin{tabular}{|c|c|}
\hline Itens & Fator 1 - Comida \\
\hline DGI1 & Consigo resistir a comer "besteiras" quando quero. \\
\hline DGI6* & Eu teria dificuldade em manter uma dieta especial e saudável. \\
\hline DGI11* & Se minha comida preferida estivesse na minha frente, eu teria dificuldade em esperar para comê-la. \\
\hline DGI16 & É fácil, para mim, resistir a doces e outras guloseimas. \\
\hline DGI21* & Às vezes eu como até passar mal. \\
\hline DGI26 & Sempre tentei me alimentar de maneira saudável, pois compensa a longo prazo. \\
\hline \multirow[t]{2}{*}{ DGI31 } & Mesmo com fome, consigo esperar até o horário da próxima refeição sem ter que comer algo. \\
\hline & Fator 2 - Realização \\
\hline DGI5 & Eu me esforcei na escola para me tornar uma pessoa melhor. \\
\hline DGI10 & Tentei me esforçar na escola para que eu pudesse ter um futuro melhor. \\
\hline DGI15* & Na escola, tentei optar pelo caminho mais fácil. \\
\hline DGI20 & Sou capaz de trabalhar arduamente para progredir na vida. \\
\hline DGI25* & Não consigo me motivar a realizar objetivos de longo prazo. \\
\hline DGI30 & Sempre senti que meus esforços seriam, um dia, recompensados. \\
\hline \multirow[t]{2}{*}{ DGI35* } & Preferiria seguir um caminho mais fácil na vida do que ter sucesso. \\
\hline & Fator 3 - Social \\
\hline DGI3* & Ao realizar alguma atividade, odeio ter de revezar com outras pessoas. \\
\hline DGI8 & Geralmente eu tento avaliar como as minhas ações afetam os outros. \\
\hline DGI13* & Penso que ajudarmos uns aos outros traz benefícios à sociedade. \\
\hline DGI18 & Tento considerar como minhas ações irão afetar outras pessoas a longo prazo. \\
\hline DGI23* & Eu não avalio como o meu comportamento afetam outras pessoas. \\
\hline DGI28 & Valorizo a necessidade das pessoas ao meu redor. \\
\hline \multirow[t]{2}{*}{ DGI33* } & Não há sentido em considerar como minhas decisões afetam outras pessoas. \\
\hline & Fator 4 - Dinheiro \\
\hline DGI4 & Quando posso, tento economizar um pouco de dinheiro em caso de emergências futuras. \\
\hline DGI9* & É difícil para mim resistir a comprar coisas que eu não posso pagar. \\
\hline DGI14 & Tento gastar meu dinheiro sabiamente. \\
\hline DGI19* & Em relação a dinheiro, não sou uma pessoa confiável. \\
\hline DGI24* & Quando eu recebo dinheiro, prefiro gastar imediatamente. \\
\hline DGI29 & Administro bem o meu dinheiro. \\
\hline \multirow[t]{2}{*}{ DGI34* } & Gosto de gastar dinheiro logo que eu ganho. \\
\hline & Fator 5 - Físico \\
\hline DGI2 & Consigo controlar meus desejos sexuais. \\
\hline DGI7 & Antes de ter uma relação sexual, prefiro conhecer melhor a pessoa. \\
\hline DGI12* & Meu hábito de me focar apenas nas "coisas boas" me causou prejuízos ao longo da vida. \\
\hline DGI17 & Eu já deixei de lado prazeres físicos ou conforto para alcançar meus objetivos. \\
\hline DGI22* & Prefiro explorar de imediato o lado físico das relações amorosas. \\
\hline DGI27* & Sempre adio cumprir uma tarefa que exige muito esforço físico. \\
\hline DGI32* & Já menti ou inventei desculpas para poder fazer algo mais prazeroso. \\
\hline
\end{tabular}

Nota. $*$ Refere-se a itens invertidos.

Psico-USF, Bragança Paulista, v. 25, n. 1, p. 75-88, jan./mar. 2020 
Sobre os autores:

Gabriel Loureiro Figueira é mestrando em Psicologia do Programa de Pós-Graduação em Psicologia da UFRJ e pesquisador no Laboratório de Psicometria e Psicologia Positiva (LP3).

ORCID: https://orcid.org/0000-0003-2265-1501

E-mail: gabrielloufi@gmail.com

Vinícius do Carmo Oliveira de Lemos é aluno de graduação em Psicologia da UFRJ e pesquisador no Laboratório de Psicometria e Psicologia Positiva (LP3).

ORCID: https://orcid.org/0000-0003-4581-9039

E-mail: lemos.vncs@gmail.com

Bruno Figueiredo Damásio é professor do Instituto de Psicologia e do Programa de Pós-Graduação em Psicologia da UFRJ, chefe do Departamento de Psicometria e coordenador do Laboratório de Psicometria e Psicologia Positiva (LP3).

ORCID: https://orcid.org/0000-0002-1150-092X

E-mail: bf.damasio@gmail.com

Contato com os autores:

Bruno Figueiredo Damásio

Instituto de Psicologia/Departamento de Psicometria - Universidade Federal do Rio de Janeiro (UFRJ)

Av. Pasteur, 250, Urca

Rio de Janeiro-RJ, Brasil

CEP: 22290-902

Telefone: (21) 3938-5331 